\title{
Research on the Optimization the Structural Parameters of Mechanical Treadmill
}

\author{
Linzhen $\mathrm{Wu}^{*}$ \\ Physical Education Department of Xi'an University of Post and Telecommunications, Xi'an 710121, China
}

\begin{abstract}
This paper proposed a three-dimensional model of treadmill, structural diagram of mechanical transmission, structural optimization model and kinematic analysis model, deriving the values of connecting rods as $0.425 \mathrm{~m}, 1.673 \mathrm{~m}$ and $0.662 \mathrm{~m}$ by solving the optimization model. It further conducts a kinematic analysis on treadmill using this set of parameters and kinematic simulation software "Motion", providing simulation curves of angular displacement, angular velocity and angular acceleration of the main moving parts such as the connecting rods, pedals and handrail handles. The simulation curves indicate that when the rotation speed of the wheels is $10 \mathrm{r} / \mathrm{min}$, the connecting rods, pedals and handrail handles move smoothly and change almost sinusoidally; the displacement of handles ranges in-700 200 mm, the speed ranges in-400 400 $\mathrm{mm} / \mathrm{s}$, the acceleration ranges in $-400 \sim 500 \mathrm{~mm} / \mathrm{s} 2$, the angular change of pedals is $-5^{\circ} \sim 30^{\circ}$, the amplitude variation of angular velocity is $<25 \%$ s and the amplitude variation of angular acceleration is $<28 \% \mathrm{~s} 2$. The above mentioned calculated prospects of the treadmill provide some reference for carrying out a quick optimization design of the treadmill.
\end{abstract}

Keywords: Kinematic analysis, Parameters optimization, Structural design, Treadmill, Three-Dimensional modeling, Structural parameters.

\section{INTRODUCTION}

Treadmill is one of the most popular fitness equipments [1-5], in which the mechanical treadmill is related to running fitness equipment used extensively in neighborhoods and on campuses because of its cheap price, simple structure and easy maintenance [6]. In addition, it is also widely used in medical rehabilitation, step rate coordinate training and in various other situations [7-10].

With the continuous development of production technology, requirements for mechanical performance and cost-effective resources are also increasing. Kinematic simulation analysis is of great significance for the improvement of treadmill performance, which is also an important method in the current treadmill design [11]. Optimization design method makes use of the computer to achieve the objective and optimizes the design of the treadmill structural parameters. If the treadmill is prepared for optimal performance, it can be conducive to marketing and sales. The optimization of mechanical treadmill is mainly based on kinematic optimization. Therefore, in order to optimize the design of the parameters of the treadmill by establishing the optimization model, three-dimensional model is created in three-dimensional software, simulating its kinematic performance through the kinematic simulation software which provides the simulation results curve, followed by analysis and research on the exercise and dynamics of the machine. The method used in this paper not only demonstrates the feasibility of the treadmill, but also provides some guidance with respect to the engineering design, finding the best solution. It can significantly

\footnotetext{
*Address correspondence to this author at the Physical Education Department of Xi'an University of Post and Telecommunications, Xi'an 710121, China; Tel: 0086-029-88166241; E-mail: wu558w9@163.com
}

can reduce the development costs, shorten the development cycle, improve work efficiency and greatly simplify the process of design and development strategies in the development of products, while improving the quality of products [12-15].

\section{THREE-DIMENSIONAL MODELING AND WORK PRINCIPLE}

The three-dimensional model of treadmill shown in Figs. (1 and 2) represents the mechanical transmission system, that is constructed by the pedals, rotary handle and wheel. When the fitnessenthusiast puts his foot on the pedals to carry out up and down movement, the wheel rotates, and this movement of the wheel passes on to the rotary handle that further induces back and forth movement. Thus, the movement of pedals and rotary handle helps in promoting the movement of the fitness enthusiast on the treadmill.

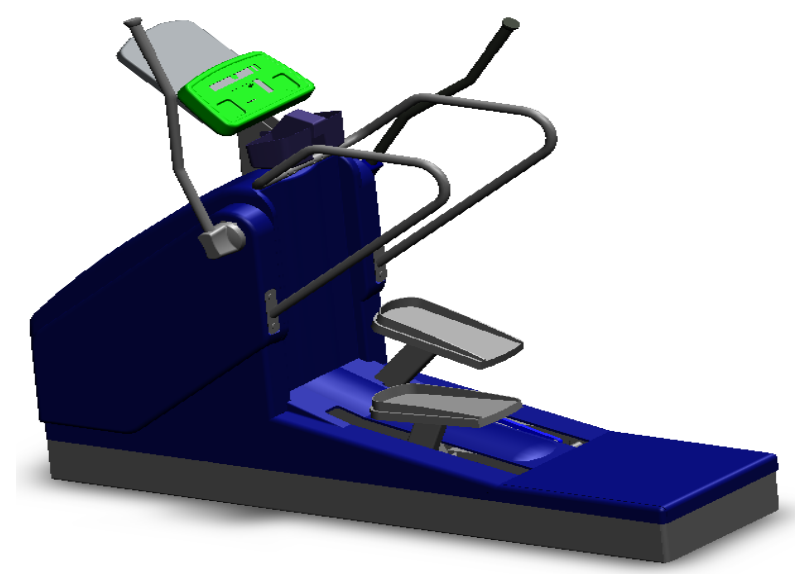

Fig. (1). Three-dimensional model of mechanical treadmill. 


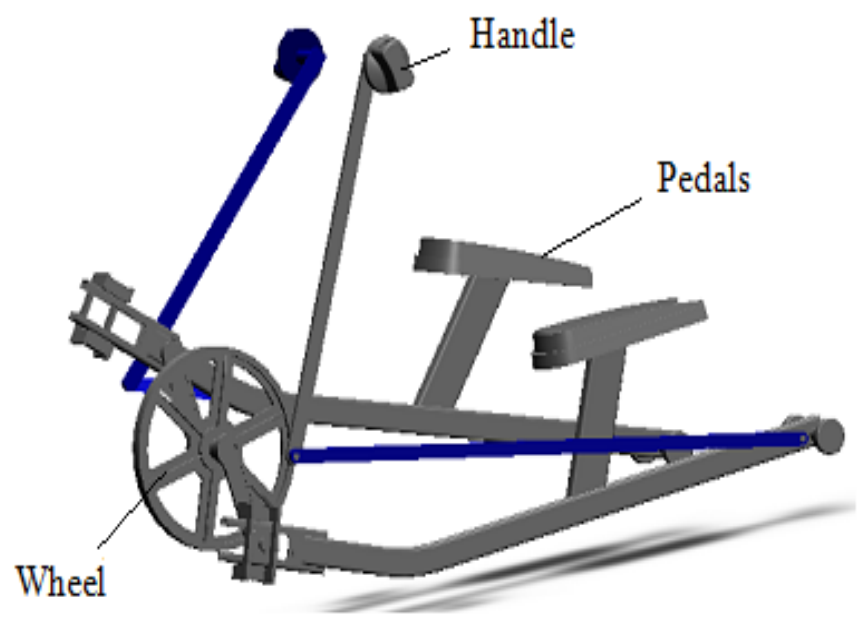

Fig. (2). Transmission system of mechanical treadmill.

\section{STRUCTURAL PARAMETERS OPTIMIZATION OF TREADMILL}

The structural parameters optimization of treadmill is based on its kinematic analysis For planar mechanisms, vector equation can be established according to a closed loop, where the projection equation of the vector equation represents the displacement equation of the mechanism; each vector equation can create two projection equations.

For a single closed chain mechanism consisting of $\mathrm{n}$ rods, the formula of closed loop vector equation is [16].

$\sum l_{i}=0$

In the formula, $l_{i}$ is the $\mathrm{i}$-th lever length, $i=1,2, \cdots, n$.

The displacement equation formula of the component can be described as:

$$
\left\{\begin{array}{l}
\sum l_{i} \cos \varphi_{i}=0 \\
\sum l_{i} \sin \varphi_{i}=0
\end{array}\right.
$$

By seeking the derivative of time $t$, the rate equation formula can be written as:

$$
\left\{\begin{array}{l}
\sum \frac{\mathrm{d} l_{i}}{\mathrm{~d} t} \cos \varphi_{i}-l_{i} \frac{\mathrm{d} \varphi_{i}}{\mathrm{~d} t} \sin \varphi_{i}=0 \\
\sum \frac{\mathrm{d} l_{i}}{\mathrm{~d} t} \sin \varphi_{i}+l_{i} \frac{\mathrm{d} \varphi_{i}}{\mathrm{~d} t} \cos \varphi_{i}=0
\end{array}\right.
$$

The acceleration equation formula is:

$$
\left\{\begin{array}{l}
\sum \frac{\mathrm{d}^{2} l_{i}}{\mathrm{~d} t^{2}} \cos \varphi_{i}-2 \frac{\mathrm{d} l_{i}}{\mathrm{~d} t} \frac{\mathrm{d} \varphi_{i}}{\mathrm{~d} t} \sin \varphi_{i}-l_{i}\left(\frac{\mathrm{d} \varphi_{i}}{\mathrm{~d} t}\right)^{2} \cos \varphi_{i}-l_{i} \frac{\mathrm{d}^{2} \varphi_{i}}{\mathrm{~d} t^{2}} \sin \varphi_{i}=0 \\
\sum \frac{\mathrm{d}^{2} l_{i}}{\mathrm{~d} t^{2}} \sin \varphi_{i}-2 \frac{\mathrm{d} l_{i}}{\mathrm{~d} t} \frac{\mathrm{d} \varphi_{i}}{\mathrm{~d} t} \cos \varphi_{i}-l_{i}\left(\frac{\mathrm{d} \varphi_{i}}{\mathrm{~d} t}\right)^{2} \sin \varphi_{i}+l_{i} \frac{\mathrm{d}^{2} \varphi_{i}}{\mathrm{~d} t^{2}} \cos \varphi_{i}=0
\end{array}\right.
$$

Assuming the unknown displacement variable $\varphi=\left(\varphi_{1}, \varphi_{2}, \cdots, \varphi_{n}\right)$, known input $q=\left(q_{1}, q_{2}, \cdots, q_{n}\right)$, thus the displacement equation is often simplified as follows

$f(\varphi, q)=0$,
$[A][\dot{\varphi}]=[B][\dot{q}]$
$[\dot{\varphi}]=\left[\dot{\varphi}_{1}, \dot{\varphi}_{2}, \cdots, \dot{\varphi}_{n}\right]^{\mathrm{T}}$

$[\dot{q}]=\left[\dot{q}_{1} \dot{q}_{2}, \cdots, \dot{q}_{n}\right]^{\mathrm{T}}$

In the formula, $[A]$ is the driven member position parameters matrix and which equals with the Jacobian matrix in form, by just changing $x_{i}$ to $\varphi_{i} ;[B]$ is the active member position of parameters matrix or matrix known parameters.

The acceleration matrix equation:

$$
[A][\ddot{\varphi}]=[B][\ddot{q}]+[B][\dot{q}]-[\dot{A}][\dot{\varphi}]
$$

Similar to the kinematic analysis of plane mechanism, the spatial kinematic analysis also includes the analysis of displacement, velocity and acceleration. In accordance with the movement relations between the active member and the driven member, the motion displacement equation simplifies to:

$$
F(\varphi, \psi)=0
$$

In the formula, $\varphi, \psi$ is respectively the angular displacement of the driven member and the active member.

By seeking the derivative of the displacement equation as described in formula (10), the velocity equation can be obtained as

$\frac{\partial F}{\partial \varphi} \dot{\varphi}+\frac{\partial F}{\partial \psi} \dot{\psi}=0$

The velocity equation is a linear equation and on solving the unknown velocity $\dot{\psi}$, and seeking its derivation, the acceleration equation can be obtained:

$\frac{\partial^{2} F}{\partial \varphi^{2}} \dot{\varphi}^{2}+\frac{\partial F}{\partial \varphi} \ddot{\varphi}+2 \frac{\partial^{2} F}{\partial \varphi \partial \psi} \dot{\psi} \dot{\varphi}+\frac{\partial^{2} F}{\partial \psi^{2}} \dot{\psi}^{2}+\frac{\partial F}{\partial \psi} \ddot{\psi}=0$

This acceleration equation is also a linear equation.

In order to optimize and analyze easily, we simplified Fig. (2) to crank rocker mechanism as shown in Fig. (3), where the design variables represent the length of each rod.

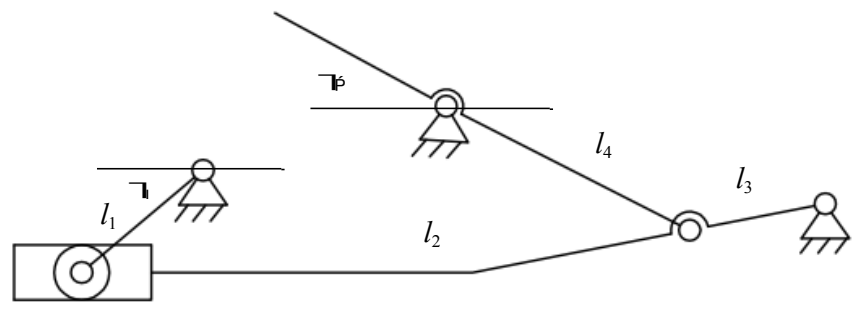

Fig. (3). Transmission system equivalent diagram of mechanical treadmill.

Assuming the function formula between the rotation angle $\theta_{i}(i=2,3,4)$ of other components and the rotation angle $\theta_{1}$ of input component,

$\theta_{i}=f\left(l_{1}, l_{2}, l_{3}, l_{4}, \theta_{1}\right)$

The velocity equation can be written as: 
In the formula, $l_{1}, l_{2}, l_{3}, l_{4}$ are transmission system connecting rod lengths of mechanical treadmill; $\theta_{1}, \theta_{2}, \theta_{3}$, $\theta_{4}$ are the intersection angles that the connecting rod is relative to the horizontal position.

Obtained by the formula (9), when the rotation angle of input component is $\theta_{1}$, the rotation angle $\theta_{i}$ of output component can be calculated by the following formula:

$\theta_{i}=\beta_{0}+\delta_{0}-\beta_{1}-\delta_{1}$

In the formula,

$\beta_{0}=\arctan \frac{l_{1} \sin \theta_{0}}{1-l_{1} \cos \theta_{0}} \delta_{0}=\arccos \frac{l_{1}^{2}-l_{2}^{2}+l_{3}^{2}+1-2 l_{1} \cos \theta_{0}}{2 l_{3} \sqrt{l_{1}^{2}+1-2 l_{1} \cos \theta_{0}}}$

$\beta_{1}=\arctan \frac{l_{1} \sin \left(\theta_{0}+\theta_{0 i}\right)}{1-l_{1} \cos \left(\theta_{0}+\theta_{0 i}\right)}$

$\delta_{1}=\arccos \frac{l_{1}^{2}-l_{2}^{2}+l_{3}^{2}+1-2 l_{1} \cos \left(\theta_{0}+\theta_{0 i}\right)}{\left.2 l_{3} \sqrt{l_{1}^{2}+1-2 l_{1} \cos \left(\theta_{0}+\theta_{0 i}\right.}\right)}$

where, $\theta_{0}$ is the initial angle of the connecting $\operatorname{rod} l_{1}$; and $\theta_{0 i}$ is the increased amount of angle of the connecting $\operatorname{rod} l_{1}$, that is $\theta_{1}=\theta_{0}+\theta_{0 i}$.

Further expression of formula (13) is [17]:

$$
\begin{aligned}
& f\left(l_{1}, l_{2}, l_{3}, l_{4}, \theta_{1}\right)=\arctan \frac{l_{1} \sin \theta_{0}}{1-l_{1} \cos \theta_{0}}+\arccos \frac{l_{1}^{2}-l_{2}^{2}+l_{3}^{2}+1-2 l_{1} \cos \theta_{0}}{2 l_{3} \sqrt{l_{1}^{2}+1-2 l_{1} \cos \theta_{0}}} \\
& -\arctan \frac{l_{1} \sin \left(\theta_{0}+\theta_{0 i}\right)}{1-l_{1} \cos \left(\theta_{0}+\theta_{0 i}\right)}-\arccos \frac{l_{1}^{2}-l_{2}^{2}+l_{3}^{2}+1-2 l_{1} \cos \left(\theta_{0}+\theta_{0 i}\right)}{2 l_{3} \sqrt{l_{1}^{2}+1-2 l_{1} \cos \left(\theta_{0}+\theta_{0 i}\right)}}
\end{aligned}
$$

It can be known from Fig. (3) that the design variables of mechanical treadmill are the length of each connecting rod; the optimization design goal of mechanism is to enable the minimum error between the rotation angle of output component and the given $\theta_{d i}$ in all positions, setting the objective function [18] as,

$F(\boldsymbol{x})=\sum_{i=1}^{3} \omega_{i}\left[f\left(l_{1}, l_{2}, l_{3}, l_{4}, \theta_{1}\right)-\theta_{d i}\right]^{2}$

where, $\omega_{i}$ is the penalty factor. The constraints of mechanism optimization design should be determined according to the actual situation of institutional design. For example, the crank rocker four-bar linkage must satisfy the following relationship [19].

$\left\{\begin{array}{l}l_{1}+l_{2} \leq l_{3}+l_{4} \\ l_{1}+l_{3} \leq l_{2}+l_{4} \\ l_{1}+l_{4} \leq l_{2}+l_{3}\end{array}\right.$

If the mechanism requires flexible and reliable transmission, then the transmission angle $\gamma$ should satisfy:

$[\gamma]_{\min } \leq \gamma \leq[\gamma]_{\max }$

Among them $\cos \gamma_{i}=\frac{l_{2}^{2}+l_{3}^{2}-l_{1}^{2}-1+2 l_{1} \cos \left(\theta_{0}+\theta_{0 i}\right)}{2 l_{2} l_{3}}$

From the above equation, the transmission angle $\gamma_{i}$ changes with the change in $\theta_{0}+\theta_{0 i}$; when $\cos \left(\theta_{0}+\theta_{0 i}\right)$ is maximum, $\gamma_{i}$ is minimum, and when $\cos \left(\theta_{0}+\theta_{0 i}\right)$ is minimum, $\gamma_{i}$ is maximum. To satisfy the above conditions, the constraint equation should be:

$\left\{\begin{array}{l}\cos [\gamma]_{\min }-\frac{l_{2}^{2}+l_{3}^{2}-l_{1}^{2}-1+2 l_{1}\left[\cos \left(\theta_{0}+\theta_{0 i}\right)\right]_{\max }}{2 l_{2} l_{3}} \geq 0 \\ \frac{l_{2}^{2}+l_{3}^{2}-l_{1}^{2}-1+2 l_{1}\left[\cos \left(\theta_{0}+\theta_{0 i}\right)\right]_{\min }}{2 l_{2} l_{3}}-\cos [\gamma]_{\text {min }} \geq 0\end{array}\right.$

The crank rocker mechanism has [20]

$\left\{\begin{array}{l}{\left[\cos \left(\theta_{0}+\theta_{0 i}\right)\right]_{\max }=1} \\ {\left[\cos \left(\theta_{0}+\theta_{0 i}\right)\right]_{\min }=-1}\end{array}\right.$

Therefore, the constraint equation is:

$\left\{\begin{array}{l}\cos [\gamma]_{\min }-\frac{l_{2}^{2}+l_{3}^{2}-\left(1-l_{1}\right)^{2}}{2 l_{2} l_{3}} \geq 0 \\ \frac{l_{2}^{2}+l_{3}^{2}-\left(1+l_{1}\right)^{2}}{2 l_{2} l_{3}}-\cos [\gamma]_{\max } \geq 0\end{array}\right.$

Assuming that the rotation angle of input component is divided into 20 equal portions, taking the right factor $\omega_{i}=1$, $l_{4}$ and other connecting rods are not in a chain link, having little impact on other parameters, then the connecting rod $l_{4}=1$ setting $\boldsymbol{x}=\left[x_{1}, x_{2}, x_{3}\right]=\left[l_{1}, l_{2}, l_{3}\right]$, thus the optimization objective function of crank link mechanism can be obtained by equation (16) and (15) as follows,

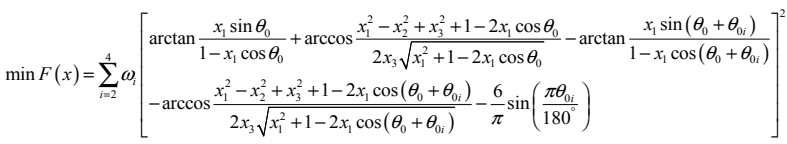

The optimization design constraints are as follows [21]

$\left\{\begin{array}{l}1-x_{1}-x_{2}+x_{3} \geq 0 \\ 1-x_{1}+x_{2}-x_{3} \geq 0 \\ -1-x_{1}+x_{2}+x_{3} \geq 0\end{array}\right.$

Required that transmission angle meets $30^{\circ} \leq \gamma \leq 135^{\circ}$, the equation (23) becomes:

$\left\{\begin{array}{l}\frac{\sqrt{3}}{2}-\frac{x_{2}^{2}+x_{3}^{2}-\left(1-x_{1}\right)^{2}}{2 x_{2} x_{3}} \geq 0 \\ \frac{x_{2}^{2}+x_{3}^{2}-\left(1-x_{1}\right)^{2}}{2 x_{2} x_{3}}-\frac{\sqrt{2}}{2} \geq 0\end{array}\right.$

According to the mechanism structure size, each component length relatively to the length of chassis is within a given size range, thus on the basis of demand, we can get 


$$
\left\{\begin{array}{l}
0.1 \leq x_{1} \leq 1 \\
1 \leq x_{2} \leq 3 \\
0.5 \leq x_{3} \leq 1
\end{array}\right.
$$

Interior point penalty function method is used for the solution. Selecting the initial penalty parameter $\gamma_{0}=0.001$, the initial point $\boldsymbol{x}=\left[x_{1}, x_{2}, x_{3}\right]=[1,3,1]$, taking the penalty function convergence precision as 0.001 , the optimal solution is:

$\boldsymbol{x}^{*}=[0.425,1.673,0.662]$

It can be seen from the optimization results that the transmission system connecting rods of mechanical treadmill are respectively $0.425 \mathrm{~m}, 1.673 \mathrm{~m}$ and $0.662 \mathrm{~m}$.

\section{KINEMATIC ANALYSIS OF MECHANICAL TREADMILL}

By using the software "Motion" to simulate the kinematics, we can obtain the displacement, velocity and acceleration parameters of the connecting rods, pedals and the handrail handles when the wheel is at rotational velocity of $10 \mathrm{r} / \mathrm{min}$. The specific simulation curves of connecting rods are shown in Figs. (4-6).

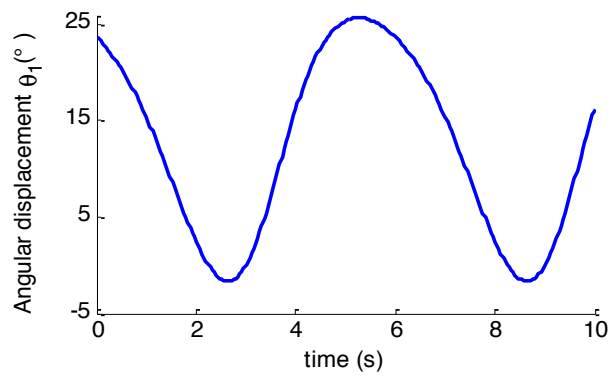

Fig. (4). The angular displacement of connecting rods.

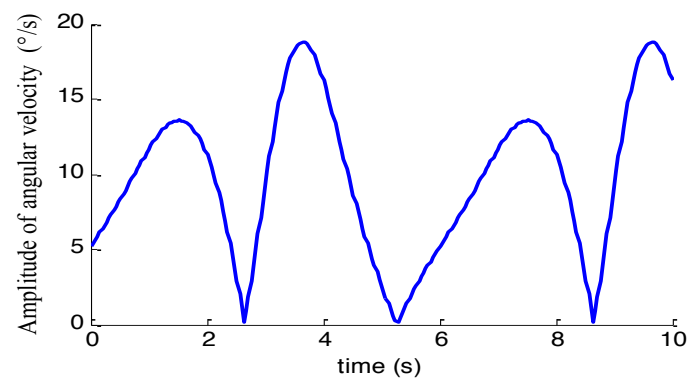

Fig. (5). The angular velocity of connecting rods.

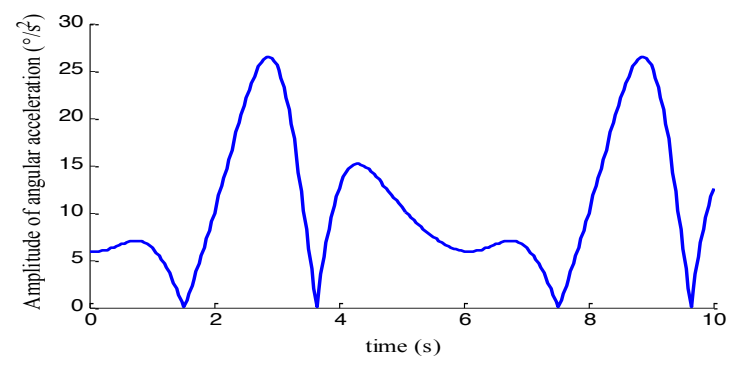

Fig. (6). The angular acceleration of connecting rods.
The simulation curves of pedals shown in Figs. (7-9).

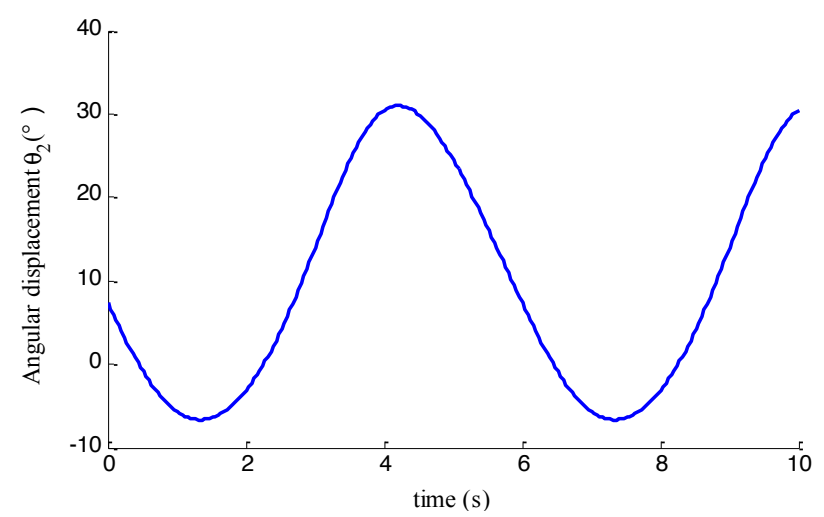

Fig. (7). The angular displacement of pedals.

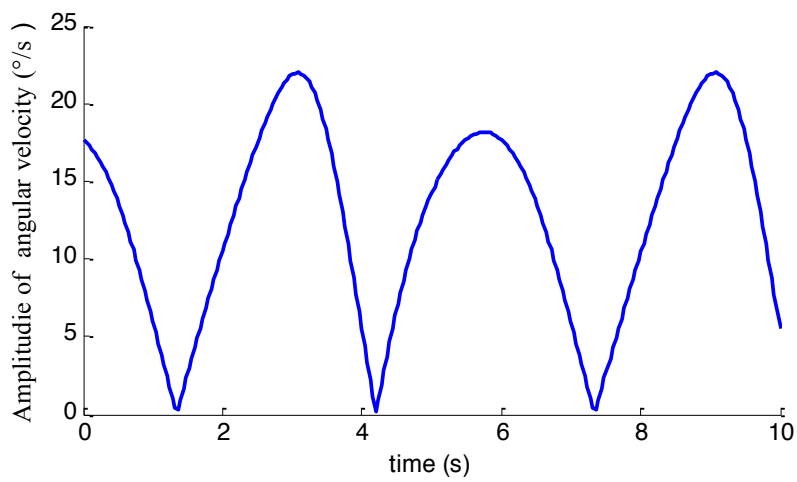

Fig. (8). The angular velocity of pedals.

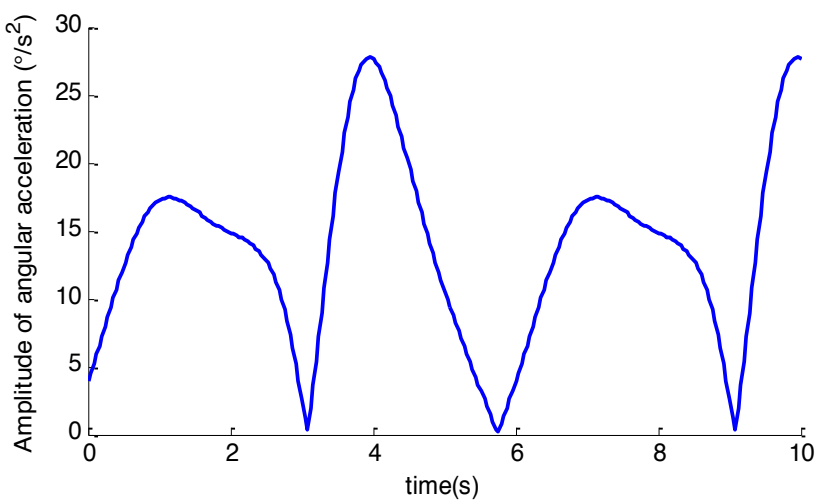

Fig. (9). The angular acceleration of pedals.

The simulation curves of handrail handles are shown in Figs. (10-12).

When the wheel is at the rotational speed of $10 \mathrm{r} / \mathrm{min}$, it can be known from the above simulation curves that the connecting rods, pedals and handrail handles motion smoothly, almost in a sinusoidal variation, and the main design parameters such as the displacement of handles are in ranges $-700 \sim 200 \mathrm{~mm}$, the velocity ranges are $-400 \sim 400$ $\mathrm{mm} / \mathrm{s}$, the acceleration ranges are $-400 \sim 500 \mathrm{~mm} / \mathrm{s} 2$, the angular change of pedals is $-5^{\circ} \sim 30^{\circ}$, the amplitude variation of angular velocity is $<25 \%$ s and the amplitude variation of angular acceleration is $<28 \%$ s 2 . This study therefore provides some reference to the quick optimization design of mechanical treadmill. 


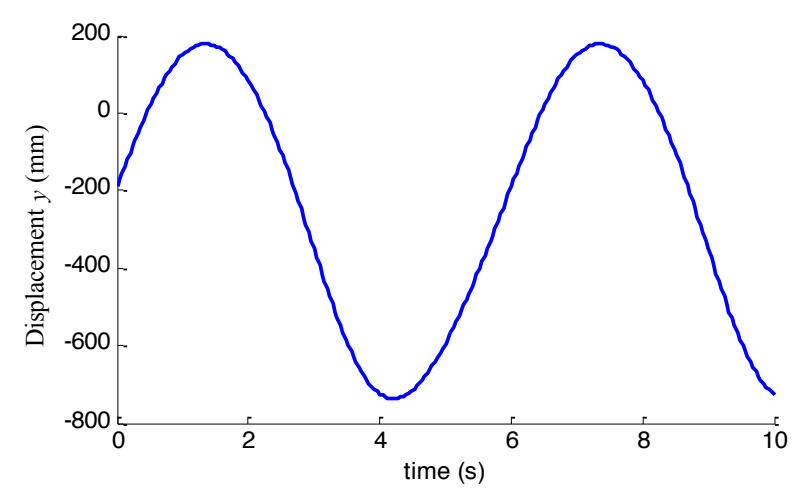

Fig. (10). The displacement of handrail handles.

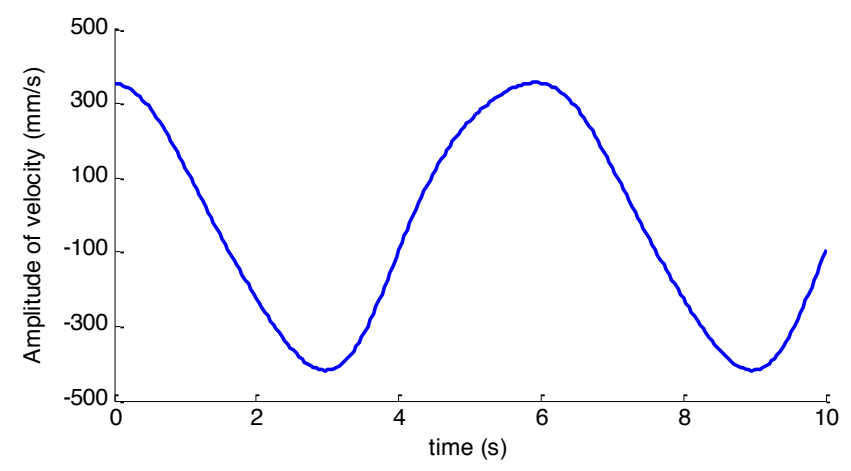

Fig. (11). The speed of handrail handles.

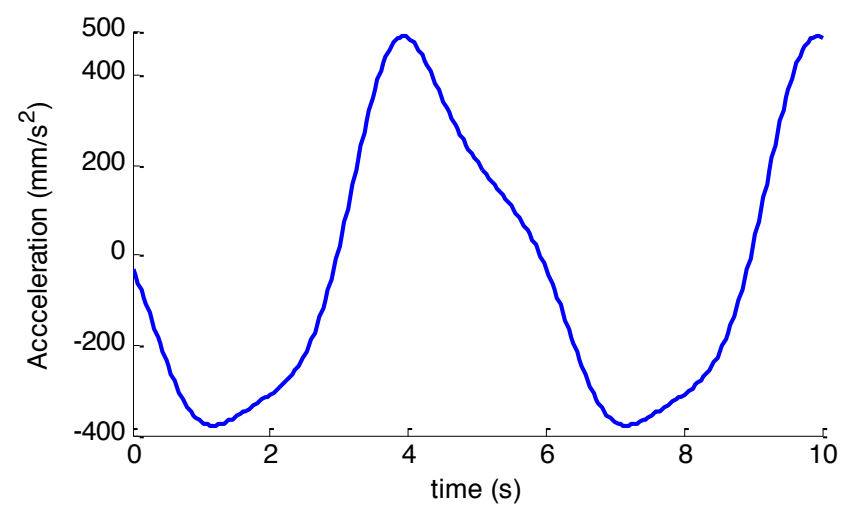

Fig. (12). The acceleration of handrail handles.

\section{CONCLUSION}

Through the parameters of optimization and kinematic simulation of mechanical treadmill, we obtained the design parameters of mechanical treadmill and the simulation curves of angular displacement, angular velocity and angular acceleration of the major moving parts, having several main conclusions as follows:

1) When the length values of connecting rods are 0.425 $\mathrm{m}, 1.673 \mathrm{~m}, 0.662 \mathrm{~m}$, and $1 \mathrm{~m}$, the treadmill gives the best performance;

2) Based on the above optimization parameters, by conducting kinematic analysis for the treadmill and extracting the simulation curves of each optimized component, it is known that the connecting rods, pedals and handrail handles motion smoothly, almost in a sinusoidal variation, and can provide the best service for to the fitness enthusiast.

3) The displacement of handles ranges are $-700 \sim 200$ $\mathrm{mm}$, the speed ranges from $-400 \sim 400 \mathrm{~mm} / \mathrm{s}$, the acceleration ranges from $-400 \sim 500 \mathrm{~mm} / \mathrm{s} 2$, the angular change of pedals is $-5^{\circ} \sim 30^{\circ}$, the amplitude variation of angular velocity is $<25 \%$ and the amplitude variation of angular acceleration is $<28 \%$ s 2 .

\section{CONFLICT OF INTEREST}

The author confirms that this article content has no conflict of interest.

\section{ACKNOWLEDGEMENTS}

Declared none.

\section{REFERENCES}

[1] X. H. Han, "Research on Speed Control System of Treadmill Digital DC," Shandong University, Jinan, 2007.

[2] J. X. Zhang, X. Q. Guo, J. R. Zhang, "Simulation of steering and driving of vehicle based on solid works and COSMOSM motion," Journal of Chongqing University of Technology, vol. 24, no. 4, pp. 13-16, 2010.

[3] M. L. Walker, S. I. Ringleb, G.C. Maihafer, R. Walker, J.R. Crouch, L.B. Van, and S. Morrison, "Virtual reality-enhanced partial body weight-supported treadmill training poststroke: feasibility and effectiveness in 6 subjects," Archives of Physical Medicine and Rehabilitation, vol. 91, no. 1, pp. 115-122, 2010.

[4] P. Reisi, S. Babri, H. Alaei, M.R. Sharifi, G. Mohaddes, S.M. Noorbakhsh, and R. Lashgari, "Treadmill running improves longterm potentiation (LTP) defects in streptozotocin-induced diabetes at dentate gyrus in rats," Pathophysiology, vol. 17, no. 1, pp. 33-36, 2010.

[5] C.S. Oza, and S.F. Giszter. "Plasticity and alterations of trunk motor cortex following spinal cord injury and non-stepping robot and treadmill training", Experimental Neurology, vol. 256, pp. 5769, 2014.

[6] H. C Steven, G. A. Peter, P. F. Daniel, and A.D. Kuo, "A simple method for calibrating force plates and force treadmills using an instrumented pole," Gait \& Posture, vol. 29, no. 1, pp. 59-64, 2009. S. Hussain, S. Q. Xie, and G. Liu, "Robot assisted treadmill training: Mechanisms and training strategies," Medical Engineering \& Physics, vol. 33, no. 5, pp. 527-533, 2011.

[8] G.J. Verkerke, A.L. Hof, W. Zijlstra, W. Ament, and G. Rakhorst, "Determining the centre of pressure during walking and running using an instrumented treadmill," Journal of Biomechanics, vol. 38, no. 9, pp. 1881-1885, 2005.

[9] S. Hesse, M. Konrad, D. Uhlenbrock, "Treadmill walking with partial body weight support versus floor walking in hemiparetic subjects," Archives of Physical Medicine and Rehabilitation, vol. 30, no. 4, pp. 421-427, 1999.

[10] A. Esquenazi, S. Lee, A. T. Packel, and L. Braitman, "A randomized comparative study of manually assisted versus roboticassisted body weight supported treadmill training in persons with a traumatic brain injury," Journal of Injury, Function, and Rehabilitation, vol. 5, no. 4, pp. 280-290, 2013.

[11] B. Mazzà, M. Donati, J. McCamley, P. Picerno, and A. Cappozzo, "An optimized Kalman filter for the estimate of trunk orientation from inertial sensors data during treadmill walking," Gait \& Posture, vol. 35, no. 1, pp. 138-142, 2012.

[12] H. Ruckstuhl, J. Kho, and M. Weed, "Comparing two devices of suspended treadmill walking by varying body unloading and Froude number," Gait \& Posture, vol. 30, no. 4, pp. 446-451, 2009.

[13] T. da Cunha Jr, P. A. Lim, H. Qureshy, H. Henson, T. Monga, and E.J. Protas, "Gait outcomes after acute stroke rehabilitation with supported treadmill ambulation training: A randomized controlled 
pilot study," Archives of Physical Medicine and Rehabilitation, vol. 83, no. 9, pp. 1258-1265, 2002.

[14] R. T. Roemmich, E. L. Stegemöller, and C. J. Hass, "Lower extremity sagittal joint moment production during split-belt treadmill walking," Journal of Biomechanics, vol. 45, no.16, pp. 2817-2521, 2012.

[15] L.H. Sloot, M.M. van der Krogt, and J. Harlaar, "Effects of adding a virtual reality environment to different modes of treadmill walking," Gait \& Posture, vol. 39, no. 3, pp. 939-945, 2014.

[16] P. Terrier, and O. Dériaz, "Persistent and anti-persistent pattern in stride-to-stride variability of treadmill walking: Influence of rhythmic auditory cueing," Human Movement Science, vol. 31, no. 6 , pp. $1585-1597,2012$.

[17] K. J. Sullivan, B. J. Knowlton, and B. H. Dobkin, "Step training with body weight support: Effect of treadmill speed and practice paradigms on poststroke locomotor recovery," Archives of Physical Medicine and Rehabilitation, vol. 83, no. 5, pp. 683-691, 2002.
[18] S. de Groot, M. Zuidgeest, and L.H.V. van der Woude, "Standardization of measuring power output during wheelchair propulsion on a treadmill: Pitfalls in a multi-center study," Medical Engineering \& Physics, vol. 28, no. 6, pp. 604-612, 2006.

[19] L. Tesio, V. Rota, C. Chessa, and L. Perucca, "The 3D path of body centre of mass during adult human walking on force treadmill," Journal of Biomechanics, vol. 43, no. 5, pp. 938-944, 2010

[20] H. Barbeau, and M. Visintin, 'Optimal outcomes obtained with body-Weight support combined with treadmill training in stroke subjects," Archives of Physical Medicine and Rehabilitatio, vol. 84, no. 10, pp. 1458-1465, 2003.

[21] M. M. LeWinter, R. L. Engler, and J. S. Karliner, "Tocainide therapy for treatment of ventricular arrhythmias: Assessment with ambulatory electrocardiographic monitoring andtreadmill exercise," The American Journal of Cardiology, vol. 45, no. 5, pp. 1045-1052, 1980.

(C) Linzhen Wu; Licensee Bentham Open.

This is an open access article licensed under the terms of the (https://creativecommons.org/licenses/by/4.0/legalcode), which permits unrestricted, non-commercial use, distribution and reproduction in any medium, provided the work is properly cited. 